Thus, the DC field very seldom exceed the action value. These attenuations can also be calculated theoretically.

Discussion Our focus was on implantable medical devices. The distance to magnetic objects was found to be critical.

\section{USE OF MOBILE PHONES AND LIGHT METRE APPLICATIONS IN THE ASSESSMENT OF THE OCCUPATIONAL LIGHTING ENVIRONMENT} 'So Young Lee*, 'Dino Pisaniello, 'Sharyn Gaskin, ${ }^{2}$ Bruno Piccoli. ${ }^{1}$ School of Public Health,
University of Adelaide, Adelaide, Australia; ${ }^{2}$ Institute of Public Health, Catholic University of
the Sacred Heart, Rome, Italy

\subsection{6/oemed-2018-ICOHabstracts. 1220}

Introduction With a variety of built-in sensors, smartphone users can do many things with only one portable device. Light metre applications measure illuminance and are offered free or at low cost. Like noise metre apps, it is tempting to use such devices for preliminary lighting surveys. However, there are few reports of studies evaluating such use, and none which have explored their potential use for blue light hazards. This paper presents preliminary data on side by side measurements of illuminance and blue light hazard (BLHF) functionweighted illuminance with a range of smartphones, apps and light sources.

Methods Phones with Android and Apple iOS operating systems and two phone apps were compared alongside a professional lux metre on a workstation desk in a mock office, set up in a dark room. A blue light filter (Hoya B440) was used directly over the sensors for the approximate BLHF weighted value.

Results The values of the illuminance and blue-weighted illuminance differed depending on distances and the types of light sources. The illuminance values for Android and Apple devices using the same software were variable suggesting differences in sensor hardware or circuitry. There were major differences between forward and rear facing sensors. The use of the blue light filter significantly reduced illuminance readings, limiting practical application for some devices.

Discussion The rationale for the use of BLHF filters on photometric instrumentation for blue light hazard assessment has been described in the literature. Calibration factors for both naked and filtered sensors need to be established for specific phones and software. The limitations and variances of particular combinations also need to be understood. However, in principle, the use of a smartphone in preliminary lighting surveys may be feasible, and if so, guidance for their use may be developed.

\section{ASSOCIATION BETWEEN ENDOCRINE FUNCTION AND RADIATION EXPOSURE}

${ }^{1,2}$ Xiuting Li*, ${ }^{2}$ Pengfei $Y u,{ }^{1}$ Haiyan Song, ${ }^{3}$ Xin Liu, ${ }^{3}$ Liangliang Zhao, ${ }^{2}$ Jun Wang, ${ }^{3}$ Dandan Yang, ${ }^{3}$ Baoli Zhu. ${ }^{1}$ Nanjing Prevention and Treatment Centre for Occupational Diseases, Nanjing 210042, China; ${ }^{2}$ School of Public Health, Nanjing Medical University, Nanjing 211166, China; ${ }^{3}$ Jiangsu Provincial Centre for Disease Control and Prevention, Nanjing 210028, China

\subsection{6/oemed-2018-ICOHabstracts. 1221}

Introduction There has been a growing focus on endocrine dysfunction especially thyroid dysfunction after radiation exposure recent years. Thyroid dysfunction caused by radiation exposure or other reasons may be associated with change in BMI, weight and even induced obesity and metabolic consequences including diabetes. On the basis of above theories, we decided to observe the likely relationship of endocrine function changes within radiation exposure.

Methods A total of 1784 subjects from physical examination organisation for occupational health were investigated. Subject information was collected with a questionnaire that was carried out through interviews in the forms of face-to-face. We entered all data into a computerised database using the statistical analysis Epidata3.1, all analyses were performed by SAS 9.1.3 software. $\mathrm{p}<0.05$ was generally accepted as statistically significant.

Results The abnormal proportion of T3 and T4 in female were higher than them in male $(p<0.05)$. Abnormal rate of FT4 increased, accompanying with the growth of age $(\mathrm{p}<0.05)$. In addition, T3 and FT3 levels may be associated with exposure time of X-ray. On the basis of this survey, abnormal rate of $\mathrm{T} 3(1.4 \%)$ and FT3 (1.0\%) were higher in group $<3$ years than that in group $\geq 3$ years $(0.3 \%$ and $0.1 \%$, respectively. $\mathrm{p}<0.05)$. We compared serum $\mathrm{T} 3, \mathrm{~T} 4$, TSH, FT3 and FT4 levels between different degrees referred to exposure time of radiation. Subjects whose duration time longer than 3 years were likely to have higher T4 contents than those who contact less than 3 years $(p<0.05)$. Furthermore, normal T4 subjects and abnormal ones were researched separately. In this study, serum T4 was significantly and positively related with BMI in the T4 normal group. Within normal T4 level, T4 was a little weak positive-correlated with BMI.

Conclusion Changes in thyroid function and glucose metabolism may appear after long time exposure to radiation.

\section{KNOWLEDGE AND BEHAVIOURS RELATED TO SUN PROTECTION AND USE SUNSCREEN AMONG ACADEMIC STAFF IN MUGLA, TURKEY}

${ }^{1} \mathrm{E}$ Tugba Alatas, ${ }^{2} \mathrm{~A}$ Kara Polat, ${ }^{1} \mathrm{G}$ Dogan, ${ }^{3} \mathrm{M}$ Picakciefe* ${ }^{*} \mathrm{MD}$. Department of Dermatology, Faculty of Medicine, Mugla Sitkı Kocman University, Mugla, Turkey; ${ }^{2}$ MD. Department of Dermatology, Istanbul Training and Research Hospital, Istanbul, Turkey; ${ }^{3} \mathrm{MD}$. Department of Public Health, Faculty of Medicine, Mugla Sitkı Kocman University, Mugla, Turkey

\subsection{6/oemed-2018-ICOHabstracts. 1222}

Introduction The increase of cutaneous melanoma, skin cancers and other skin diseases incidence is parallel with the increase in sun exposure. The aim of this study was to investigate the knowledge, attitudes and behaviours of academical staff in Mugla in Turkey related to sun protection.

Methods A cross sectional analysis has been conducted in which 1437 academic staff were selected. Of this selected group, 438 participated in the study. Academic staff in Mugla were asked to complete our questionnaire consisting of 26 questions. The questionnaire included questions about the socio-demographic characteristics of the participants, the stories of sunburn and skin cancer, attitudes and behaviours related to sun protection, the habits of using sun protective creams, and the levels of knowledge about sunscreen creams and ultraviolet.

Results The study was composed of 196 (44.7\%) women, 242 (55.3\%). Mean age was 38.6 \pm 0.3 . Using, using sunglasses, avoiding sunlight were among the first three methods of sun protection practiced by the academic staff. $36.1 \%$ of the participants stated that they only used sun protection cream while 'only going to sea', $29.5 \%$ of the participants stated that they used 'only in summer' and $14.6 \%$ of the participants stated that they always used. When academic staff assessed 
knowledge levels of sunscreen creams, sun protection factor (SPF), ultraviolet A (UVA) and ultraviolet B (UVB), it was determined that $11.2 \%$ of the participants knew the definition of SPF 30. In the question about UVA and UVB, 27.9\% answered correctly.

Discussion The use of sun protective cream among the sun protection methods among the academic staff was found to be the first place. However, it has been determined that this method of protection is not conscious and orderly. Academic staff's knowledge of sunscreen creams, SPF, UVB and UVA was found to be inadequate. Therefore, we believe that future sun protection programmes could target in order to improve academic staff's knowledge and attitudes towards sun protection, effects of the sun on skin and skin cancer.

\section{OCCUPATIONAL RADIATION EXPOSURE TO PREGNANT HEALTHCARE WORKERS}

Hassan A AlGhamdi, Moazzam Zaidi*, Ezzat Mansour, Hussam Saeed. Johns Hopkins Aramco Healthcare (JHAH). ${ }^{2} \mathrm{HHAH}$

\subsection{6/oemed-2018-ICOHabstracts. 1223}

Introduction Radiation exposure to healthcare workers is a known occupational hazard. With a significant number of female healthcare employees of childbearing age in the workforce, it is essential to ensure that occupational exposure is at the lowest possible limits. The fetus is sensitive to high doses of ionising radiation. The probability that a particular effect will occur depends on several factors, including dose, stage of gestation, and dose rate. A number of strategies and administrative controls are used to protect healthcare workers.

Method JHAH has an active radiation protection program for pregnant radiation workers. The program includes training, exposure monitoring and record review, modifications to working conditions, protective devices and administrative procedures to control exposure. The JHAH Radiation Protection Team provides individual case-based risk assessments and, if required, work condition modifications and necessary administrative procedures are discussed. In addition, occupational medicine provides advice and counselling as required. Two case studies will be discussed showing how the JHAH Radiation Protection program works.

Results Assessments have shown effective use of time, distance and shielding resulted in exposures well below advised guidelines. Additional radiation monitors worn at the abdomen level of pregnant healthcare workers resulted in enhanced fetus monitoring. In cases assessed, readings have been found below the level of public exposure.

Discussion/conclusion The JHAH Radiation Protection program demonstrates that proactive measures and regular monitoring provides healthcare workers with reassurance that exposure is well below exposure limits. Risk assessment for pregnant healthcare workers at work provides further reassurance that employees and their fetuses are safe. This psychological assurance has made a positive contribution to increased morale and satisfaction of the healthcare workers.

It is important to establish radiation guidelines and training programs for pregnant radiation healthcare workers.
1283 GENE EXPRESSION ANALYSIS OF BLOOD CELLS IN RADIATION HEALTH CARE WORKERS OCCUPATIONALLY EXPOSED TO IONISING RADIATION

${ }^{1} \mathrm{R}$ Brugaletta ${ }^{*},{ }^{1,2} \mathrm{~A}$ Santoro, ${ }^{3} \mathrm{~A}$ Alisi, ${ }^{3} \mathrm{~N}$ Panera, ${ }^{4} \mathrm{~A}$ Pastore, ${ }^{4} \mathrm{G}$ Di Giovamberardino, 1,2MR Vinci, 1,2V Camisa, 'L Lavorato, ${ }^{1,2} 2 \mathrm{~S}$ Zaffina. ${ }^{1}$ Health Technology Assessment and Safety Research Unit, Bambino Gesù Children's Hospital IRCCS, Rome, Italy; ${ }^{2}$ Occupational Health Service, Bambino Gesù Children's Hospital IRCCS, Rome, Italy; ${ }^{3}$ Liver Research Unit, Bambino Gesù Children's Hospital IRCCS, Rome, Italy; ${ }^{4}$ Research Genetics and Rare Diseases Unit, Bambino Gesù Children's Hospital IRCCS, Rome, Italy

\subsection{6/oemed-2018-ICOHabstracts.1224}

Introduction Ionising radiation (IR) is a ubiquitous environmental agent whose effects on organism are well established. A direct interaction of IR with human cells causes a variety of biological effects including neoplastic transformation. In this context, physical and biological dosimetry could be a key tool for individual monitoring of the absorbed IR. However, since the current dosimetry approaches are not tailored to distinguish the early response and long-term pro-oncogenic effects of low-dose (LD)IR the discovery of inherent biomarkers represents a priority area. We hypothesised that changes of gene expression profiles in blood cells may occur during occupational exposure to LDIR and that the identification of these changes could be potentially useful in the early recognition of pro-oncogenic phenotypes.

Methods We included 9 workers exposed to IR and 3 nonexposed workers, all equipped with dosimeter. Exclusion criteria were smoking, history of cancer diagnosis before occupational exposure, high blood pressure medication and intake of paracetamol. Blood cells were collected from each worker and used to evaluate transcriptional expression of a panel of 624 cancerrelated genes by using the Real-Time PCR OpenArray technology.

Results The analysis in radiation workers exposed showed the over-expression of some genes involved in the progression of certain types of tumours and an evident alteration in the expression of the gene involved in a way of regulation, known to predispose to genomic instability and tumorigenesis induced by IR. Intriguingly, we also found that among radiation workers 3 subjects, with occupationally recognised thyroid cancer displayed an up-regulation of the rearranged during transfection (RET) gene, which has been found previously overexpressed in subjects with radiation-induced thyroid cancers.

Discussion Our preliminary data would indicate that exposure to LDIR is able to alter the expression of pro-tumour genes in blood cells, posing questions and opening new scenarios towards a 'personalised radioprotection' model.

\section{GENOTOXIC DAMAGE AND DNA REPAIR GENE POLYMORPHISMS IN WORKERS EXPOSED TO LOW DOSES OF IONISING RADIATION}

${ }^{1} \mathrm{P}$ Lovreglio*, ${ }^{1} \mathrm{R}$ Rotondi, ${ }^{1} \mathrm{P}$ Chiarappa, ${ }^{2} \mathrm{~N}$ Resta, ${ }^{2} \mathrm{R}$ Bagnulo, ${ }^{1} \mathrm{I}$ Drago, ${ }^{3} \mathrm{ME}$ Fracasso, ${ }^{3} \mathrm{D}$ Doria, ${ }^{1} \mathrm{~L}$ Soleo. ${ }^{1}$ Interdisciplinary Department of Medicine, Section of Occupational Medicine 'E.C. Vigliani', University of Bari, Italy; ${ }^{2}$ Department of Biomedical Sciences and Human Oncology, Division of Medical Genetics, University of Bari, Italy; ${ }^{3}$ Department of Medicine and Public Health, Section of Pharmacology, University of Verona, Italy

10.1136/oemed-2018-ICOHabstracts. 1225 